\title{
Algunas observaciones sobre la evolucion del lenguaje médico a través de los tiempos
}

\author{
Walter Ledermann D.
}

\section{Some remarks on the evolution of medical language across the times}

The language employed by the physicians in their communications has been suffering a continuous evolution through the times, from the sober beauty that in their conciseness and accuracy had the Greek and the Latin, to the verbosity and flowery of the Middle Age and posterior centuries, for ended in the poverty and monotony characteristics of the today publications. A brief selection of different papers and book's extracts is presented in order to illustrate these changes, including words of Hippocrates, Celsus, Leonardo, Van Leeunwenhoek, Spallanzani, Jenner, Koch, Laveran, Manson, Grassi and other less conspicuous, ending with the assertion that the scarce time for read adduced by physicians must not serves like an excuse for ignorance and lack of culture.

Key words: Medical language, evolution, writing.

Palabras clave: Lenguaje médico, evolución, escritura.

\section{Laboratorio de Microbiología, Hospital Luis Calvo Mackenna.}

Recibido: 28 de diciembre de 2010 Aceptado: 4 de enero de 2011

Correspondencia a: Walter Ledermann oncemayor@gmail.com
$\mathrm{E}$ 1 lenguaje que los médicos empleamos para comunicar nuestros descubrimientos y experiencias ha sufrido una continua evolución a lo largo del tiempo, desde la sobria belleza que en su concisión y brevedad tenían el griego y el latín, a la ampulosidad y florituras de la Edad Media y de los siglos posteriores, para terminar en la pobreza y monotonía abrumadoras que caracterizan nuestras actuales publicaciones.

En la Antigüedad no había experimentación y, no habiendo hallazgos que comunicar, no existían publicaciones similares a las revistas médicas, contentándose los maestros de entonces con exponer la experiencia de toda una vida en algún texto de enseñanza. En ellos el lenguaje llegó a alcanzar extremos de belleza inigualados, fundiéndose la medicina en la filosofía, como puede apreciarse en los aforismos de Hipócrates, en el siglo V o IV AC:

La vida es corta, el arte largo; la oportunidad fugaz, la experimentación peligrosa, el juicio dificultoso. No basta al médico hacer lo necesario; paciente y asistente deben hacer igualmente su parte y las circunstancias ser favorables... ${ }^{1}$ ¡Todo el ejercicio de la medicina, con el azar y la incertidumbre que conlleva, expresado con una brevedad y perfección deslumbrantes! Y conste que con la traducción se pierde mucho, pues hay que echar mano al doble de las palabras requeridas en la lengua original. Y, para no abrumar con el famoso juramento y otros aforismos, esta observación que se adelanta a su tiempo, pleno de supersticiones y castigos divinos: Cada enfermedad tiene una causa natural y nada ocurre sin una causa natural...

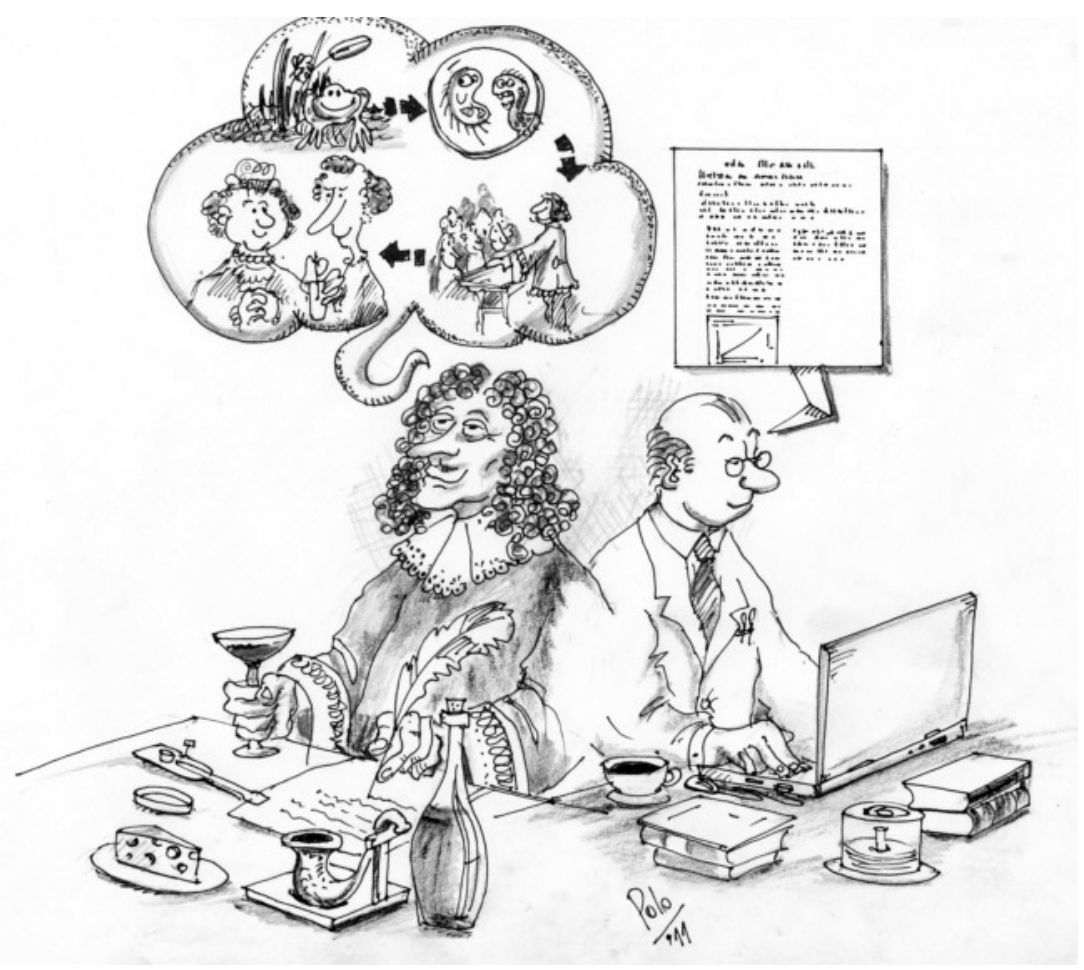

Hoy en día el lenguaje predominante, neutro -norteamericano-insípido e impersonal, ha prohibido el uso de cualquier expresión personal, empezando por la primera persona. En la Antigua Roma, maestros como Celso y Galeno no evitaban su uso, y así dice el primero: Yo, por mi parte estimo, en cuanto a las pociones medicinales $y$ 
los lavados, que no deben ser administrados sino rara vez...2. Veintidós palabras en la traducción, contra trece en el original : Ego autem medicamentorum dari pociones, et alvum duci non nisi raro debere, concedo. ¡Y se quejaba sobre la penuria e insuficiencia del vocabulario y tecnicismo de la lengua latina para designar determinadas dolencias! ${ }^{3}$. Y, otra blasfemia para los códigos actuales, Celso no desdeñaba el lenguaje coloquial ni la anécdota en medio de la ciencia: Oigo a cada paso decir que quien come una cría de golondrinas se verá todo el año libre de anginas ... ${ }^{2}$.

Suele pensarse que la Edad Media, con su apagón cultural, empobreció la expresión, que se hizo más lata y florida pero de poca sustancia, y así se desprende de la lectura de numerosos textos, pero de pronto se encuentran algunas perlas, como estos aforismos del médico $-\mathrm{y}$ también filósofo- judío Isaac Israeli, siglos IX y X, que no desmerecen ante los de Hipócrates :

La mayoría de las enfermedades curan sin la ayuda del médico mediante la ayuda de la Naturaleza...Si no puedes curar al paciente mediante la dieta, no te vuelvas a las drogas ...Siempre haz sentir al paciente que curará, aun cuando no estés convencido de ello, porque ayuda al efecto sanador de la Naturaleza... ${ }^{4}$.

El Renacimiento no sólo significó una apertura en el arte sino también en las rudimentarias ciencias de entonces. El mismo Leonardo Da Vinci hizo ocultas disecciones que dejaron al descubierto los errores anatómicos de Galeno: la investigación médica, prohibida y herética, nacía en el secreto. Deslumbrado por la maravilla de la máquina humana, concluye: Aunque el ingenio humano pueda crear múltiples inventos, nunca diseñará ninguno más hermoso, más sencillo ni más adecuado a su fin que la naturaleza, porque en sus inventos no falta ni hay nada superfluo...5.

Pronto los investigadores, distantes pero unidos por una misma llama, comenzarán a buscarse y a fundar sociedades esotéricas, comunicándose por cartas sus descubrimientos, comunicaciones que constituyen el embrión de la revista científica. En ellas el autor no evitaba sus datos personales y mencionaba tranquilamente su casa y, más tarde, ya desarrollada la medicina, su consulta.

Revisando al azar en "mi biblioteca", como se decía antaño, y no "una revisión aleatoria de antiguas publicaciones", como se diría hoy, he seleccionado al azar algunos textos, no todos del mismo valor en cuanto a su significación en la historia de la medicina, pues la selección se ha hecho por la forma y no por el fondo.

En 1672 escribe Antoine van Leeuwenhoek, el padre de la microscopia, en una de sus comunicaciones a la Royal Society: He tenido en mi casa a varias señoras muy interesadas en ver las pequeñas anguílulas del vinagre. A ellas les gustó tan poco el espectáculo, que prometieron no volver a utilizar el vinagre. Pero... ¿qué pasaría si le dijese a esta gente que en la saliva, que cubre los dientes de la boca de un hombre, están viviendo más animales que hombres sobre un reino?... Ahora, en una introducción, el célebre óptico diría: "La visión de las anguílulas puede desagradar al público"6.

Saltando un siglo, en 1786, Spallanzani llama al pan, pan, y al vino, vino: Llamo verde a la rana de que voy a hablar, porque la parte superior de su cuerpo está teñida de ese color de una manera más o menos marcada; y la llamo acuática porque habita en las aguas, sobre todo en las de los pantanos donde crece el arroz y en las de las zanjas; la distingo, pues, de las ranas que habitan en tierra y de las que se encuentran en los árboles ${ }^{7}$. Notable ejemplo de lenguaje preciso y sobrio, pero hoy día el mismo Spallanzani, luego de citar los nombres científicos de ambas ranas en cursiva, diría apenas que difieren en habitat.

Una década después, en 1796, escribe Jenner, con soberbia elegancia: El alejamiento del hombre del ambiente en el cual fuera colocado originariamente por la naturaleza, ha resultado para él una fuente fecunda de enfermedades. Su amor al boato, su complacencia en el lujo y su afición a las diversiones, lo han familiarizado con un gran número de animales que no estaban destinados a vivir en contacto con él. El lobo, despojado de su ferocidad, descansa ahora en el regazo de las damas ( Mr. John Hunter probó experimentalmente que el perro no es sino el lobo en un estado de degeneración); el gato, el pequeño tigre de nuestra isla, cuyo albergue natural es el bosque, también está domesticado y es igualmente acariciado... ${ }^{8}$. En 2011 DC, el Comité Editorial de Lancet aconsejaría al Dr. Jenner reducir esta florida introducción a una frase: "La progresiva convivencia del hombre con animales domésticos ha generado nuevas entidades nosológicas." Al terminar su Primera Memoria, Jenner anticipa, con mucha modestia, la frase clásica con que terminan hoy muchos artículos: mayores estudios son necesarios... Dice Jenner: hay en lo expuesto, cierto que ocasionalmente, algunas conjeturas expresamente destinadas para presentar, a la consideración de los que están capacitados para tales discusiones, temas apropiados para una investigación más precisa ${ }^{9}$.

Avanzando al siglo XIX, en esta frase del Dr. Hodgson, en 1819, encontramos un dramatismo que hoy chocaría a los editores de cualquier tratado médico y que quizás repugnaría a El Mercurio usar en su crónica policial: Un hombre, que habia regresado hace poco de la Jamaica, donde había sido cruelmente atormentado de la disentería, fue atacado por una neumonía violenta que, en cinco días, le condujo a la tumba ${ }^{10}$.

No le va en zaga, en el mismo siglo, año 1865, el célebre profesor Trousseau al describir un caso de coqueluche: Hace más de veinticinco años, fui llamado a una casa de la calle Chaussée-d'Antin, por una señorita, natural de 
Bordeaux, quien, me contaba su padre, había cogido en viaje un reuma violento. Esta enferma llegó a París con una fiebre vehemente, y su tos no le dejaba tregua ni de día ni de noche ${ }^{11}$. Indudablemente, Ralph Feigin no le confiaría el capítulo de Pertussis a un autor tan abundante en detalles domésticos.

Y admirable la ligereza y soltura del sabio Koch en la página 221 del Berliner Klinischen Wochenschrift del 10 abril de 1882, al hablar de una cantidad, sin molestarse en precisar el número: Cuarto experimento. Se inoculó una cantidad de animales -ratones, ratas, erizos, un criceto o hamster, palomas, sapos-cuya susceptibilidad a la tuberculosis aún no se conocía, con un cultivo obtenido del pulmón tuberculoso de un mono y cultivado fuera del organismo del animal durante ciento trece dias ${ }^{12}$. ¿Qué diría hoy el editor del $N$ Engl J Med ante la imprecisión de este "Material y métodos"?

Siguiendo con el toque personal, entramos ahora en una ácida controversia, en que los rivales son señalados con pelos y señales. Escribe Laveran, en 1891: Machiafava y Celli han elevado, a la luz del descubrimiento de estos elementos, reivindicaciones sobre las cuales habré de volver cuando analice los trabajos posteriores a los mios; estos observadores han pretendido claramente que yo no he visto más que las formas pigmentadas más gruesas de los cuerpos esféricos y que yo no he descrito los movimientos ameboides; yo creo mi deber reproducir al respecto algunos pasajes de mis primeras publicaciones ${ }^{13}$. Los italianos evitaron cuanto pudieron responder a la provocación, reservando su virulencia para los ingleses; a fin de comparar caracteres y lenguajes entre estos dos pueblos, revisemos las respectivas "introducciones" en los más célebres textos de dos famosos rivales.

Grassi comienza la Introduzione de su obra capital con el caballeroso reconocimiento a un amigo, como se estilaba en esa época: Como me decía el amigo, honorable Giustino Fortunato, al término de la primera vez que he tenido el honor de encontrarme con él, la malaria constituye el problema esencial para la Italia... En cuanto a Ross, su rival británico, le aplica toda la fuerza del lenguaje en varios párrafos: los experimentos de Ross no eran acuciosos, Ross refiere para su capote la historia del descubrimiento según su modo de ver...; y así sigue ${ }^{14}$. Lo que respondiera Ross puede imaginarse, pero para comparaciones de caracteres étnicos, vayamos al maestro de Ross, el famosísimo Patrick Manson, con su autorreferente inicio de una de sus mayores obras : $L a$ preparación exhibida a la Sociedad el 17 de Mayo por el doctor Thin, como un ejemplo de la enfermedad que yo he llamado escroto linfático, es única...yo creo que esta es la primera instancia en la cual el parásito ha sido encontrado en su propio habitat, un vaso linfático, y ha sido visto in situ. Hay dos "yo" en el primer párrafo, tres en la página siguiente, cuatro en la otra and so on ${ }^{15}$.
¿Y cómo andamos por casa ? En Chile se seguía la línea europea del lenguaje dramático del profesor Trousseau. En 1894 el doctor Monreal escribe en El Progreso Médico este artículo "original": Encontrándome en la dispensería oculística del Dr. Barrenechea el día 16 de Noviembre del año próximo pasado, tuve ocasión de ver i observar un caso, único hasta entonces presentado alli de Quiste de equinococcus de la órbita, i la casualidad quiso que poco después, el 6 de Marzo del presente año, se presentase un segundo enfermo de esta rara afección ${ }^{16}$. ¿ Rechazaría nuestro editor un "Caso clínico" que empezara : "Me tocó turno en la noche de Navidad de 1995, en la UCI de la Clínica Las Condes, donde llegó un señor de Cauquenes, terriblemente angustiado"?

Más dramático todavía es el lenguaje usado por del doctor Federico Puga Borne en su clásico texto Latrodectus, que se editara en 1892: Era en una hacienda próxima a Chillán, mi pueblo natal; una anciana que trabajaba en la emparva de una sementera de trigo habia sido picada por la araña, i sus compañeros la habían conducido a "las casas" en busca de socorro. Los alaridos incesantes i desesperados de la enferma, la emoción i la angustia pintada en todos los rostros i la agitación afanosa de la familia i la servidumbre, fué todo lo que yo pude ver $i$ oir... ${ }^{17}$. Si escribiera su libro ahora, el investigador quizás pondría este párrafo como epígrafe, en letra pequeñita, debajo del título y antes de iniciar el texto propiamente tal

Volviendo a Europa, ya en 1912, en pleno siglo XX, esta curiosidad. En una revista de filantropía, dice Paul Strauss, con el lenguaje austero que comenzaba a despuntar: De acuerdo a la estadística más reciente del servicio de protección a la primera edad, 90.146 infantes han sido colocados con nodrizas en 1906. De este número, 61.899 son niños legítimos y 28.247 niños ilegítimos, esto es, una proporción de 313 ilegítimos por mil niños. La estadística demuestra el hecho que la proporción de niños alimentados exclusivamente al pecho (30 porcien, aproximadamente, y tanto como los registros obtenidos permiten afirmarlo) ha disminuido sensiblemente en los últimos años ${ }^{18}$. Esta clasificación en legítimos e ilegítimos es muy, pero muy actual...¿O acaso no les recuerda a los caucásicos y a los hispánicos de las revistas norteamericanas del siglo XXI?

El avance es inexorable; el personalismo, el colorido y coloquio se baten retirada. Ya en 1930 y nada menos que en Francia, paradigma del lenguaje elegante, comienza a aparecer el lenguaje sobrio, "científico", que terminará por imponerse a la dramática locuacidad del profesor Trousseau. Dice el sabio Besredka, presentando un caso clínico sin ningún sensacionalismo y con la mayor precisión: A.,21 años; el comienzo de la rinitis atrófica se remonta a tres años. al examen: atrofia de los cornetes medios, exudado en los inferiores; costras en los meatos, obstruyendo casi por completo las fosas nasales y des- 
prendiendo un olor muy fétido. A la siembra: bacilos de Abel-Lowenberg ${ }^{19}$.

De aquí en adelante, con el correr de los años y la multiplicación infinita de las publicaciones, la expresión se ha venido haciendo necesariamente más sucinta y precisa, terminando en la aceptación universal de un estilo seco y anodino impuesto por los norteamericanos, con esas frases estándar que suelen arrancarnos una sonrisa, como ese remache final a la investigación fracasada, requiriendo nuevos y mayores estudios sobre el problema. Y así, de la economía y sencillez de griegos y romanos, hemos pasado, a través de los siglos, a la parquedad y pobreza del lenguaje actual, que evita cuidadosamente toda referencia personal y que admite, a veces, la primera persona del plural, aunque el autor sea uno solo, pero jamás la primera del singular.

¿Puede revertirse esta situación? Nos parece..., es decir, me parece, que no hay remedio. El número de publicaciones es infinito, los especialistas tenemos que administrar con usura el tiempo dedicado a su revisión, y terminamos muchas veces con la lectura del resumen, cuando no del puro título. Y si es escaso el tiempo que los médicos pueden dedicar a la literatura científica, mal pueden dedicar una hora a la verdadera literatura. Entonces, los médicos no leen y, por ende, no escriben -me refiero a "escribir de verdad"- pero temo que la frasecita manida de "no tengo tiempo para leer" sea apenas una mala excusa para la ignorancia y la incultura.

\section{Resumen}

El lenguaje que empleamos los médicos en artículos y libros ha sufrido una continua evolución a lo largo del tiempo, desde la sobria belleza que en su concisión y brevedad tenían el griego y el latín, pasando por la ampulosidad y florituras de la Edad Media y de los siglos posteriores, para terminar en la pobreza y monotonía que caracterizan nuestras actuales publicaciones. Una breve selección formal de textos de diferentes épocas, incluyendo palabras de Hipócrates, Celso, Leonardo, Van Leeunwenhoek, Spallanzani, Jenner, Koch, Laveran, Manson, Grassi, y otros menos conspicuos, concluye en que, si bien el tiempo que los médicos tenemos para leer es escaso, ello no debe servir como excusa para la ignorancia y la incultura.

\section{Referencias}

1.- Chadwick J. Hippocrates. En: Collier's Encyclopedia. Crowell Collier and Mac Millan, Inc, USA 1967; 12 : 135-8.

2.- Celso A.C. Los ocho libros de la medicina. Editorial Iberia S.A., Barcelona 1966. I: 111.

3.- Blánquez A. Aurelio Celso y sus ocho libros "de medicina". En: Celso A.C. Los ocho libros de la medicina. Editorial Iberia S.A., Barcelona 1966; I: XI.

4.- Heynick F. Jews and medicine. An epic saga. KTAV Publishing House, Inc. NJ 2002; p. 93.

5.- Da Vinci, L. Cuadernos. Edición de H. Anna Suh. Parragon Books Ltd., Bath 2006; p. 150.

6.- Proceedings, Royal Society, London 1672. En : Dobell C. Antony van Leeuwenhoek and his "little animals". Staples Press, London 1932; p. 55.

7.- Spallanzani, L. Experiencias para servir a la historia de la generación de animales y plantas. Memoria Primera, De la generación de algunos animales anfibios. I. Historia de la generación de la rana acuática verde. Emecé Editores, S.A. Buenos Aires 1945; p. 13.

8.- Jenner, E. Estudios sobre las causas y efectos de la vacuna variólica o cow-pox, 1798. En : Eduardo Jenner. Las tres memorias originales sobre la vacunación antivariólica. Emecé Editores, S.A., Buenos Aires 1946; p. 19.

9.- Ibid, p. 72.

10.- Hodgson, J. Traité des maladies des artères et des veins. Chez Anselmo Gabon, Libraire, Montpellier 1819 ; I: 5.

11.- Trousseau, A. Clinique Medicale de L'Hôtel Dieu de Paris, JB Balliére et fils, Paris 1865, II: 414.

12.- Koch, R. La etiología de la tuberculosis y otros trabajos. Editorial Universitaria de Buenos Aires, Buenos Aires 1965; p. 71.

13.- Laveran, A. Du paludisme et de son hématozoaire, G. Masson, Editeur, Libraire de L'Académie de Médecine, Paris $1891 ;$ p. 18.

14.- Grassi, B. Studi di uno zoologo sulla malaria. Giunti Gruppo Editoriale, Firenze 1998; pp: 83, 123, 125.

15.- Manson, P. The Filaria sanguinis hominis : and certain new forms of parasitic disease in India, China and warm countries (1883). H.K. Lewis 136, Gower Street, W.C., London 1883; pp: 1-5.

16.- Monreal Marín, J.-Contribución al estudio de los quistes de equinococcus de la órbita. El Progreso Médico. Enero 1894; IV (1): 1-18.

17.- Puga Borne, F. Latrodectus formidabilis de Chile. Imprenta Cervantes, Calle de la bandera 73, Santiago 1892; p.3

18.- Strauss, P. Les causes de la mortalité. Rapport general presenté a la Commission de la Dépopulation. Revue Philanthropique, XXXI: 122.

19.- Besredka, A. Antivirus thérapie. Masson \& Cie, Editeurs, Paris; p. 96. 